\title{
touché Is Required for Touch-Evoked Generator Potentials within Vertebrate Sensory Neurons
}

\author{
Sean E. Low, ${ }^{1}$ Joel Ryan, ${ }^{1}$ Shawn M. Sprague, ${ }^{2}$ Hiromi Hirata, ${ }^{2}$ Wilson W. Cui, ${ }^{2}$ Weibin Zhou, ${ }^{2}$ Richard I. Hume,${ }^{2}$ \\ John Y. Kuwada, ${ }^{2}$ and Louis Saint-Amant ${ }^{1}$ \\ ${ }^{1}$ Département de Pathologie et Biologie Cellulaire, Groupe de Recherche sur le Système Nerveux Central, Centre d'Excellence en Neuromique de l'Université \\ de Montréal, Université de Montréal, Montréal, Québec H3T 1J4, Canada, and ²Department of Molecular, Cellular and Developmental Biology, University of \\ Michigan, Ann Arbor, Michigan 48109-1048
}

\begin{abstract}
The process by which light touch in vertebrates is transformed into an electrical response in cutaneous mechanosensitive neurons is a largely unresolved question. To address this question we undertook a forward genetic screen in zebrafish (Danio rerio) to identify mutants exhibiting abnormal touch-evoked behaviors, despite the presence of sensory neurons and peripheral neurites. One family, subsequently named touché, was found to harbor a recessive mutation which produced offspring that were unresponsive to light touch, but responded to a variety of other sensory stimuli. The optogenetic activation of motor behaviors by touché mutant sensory neurons expressing channelrhodopsin-2 suggested that the synaptic output of sensory neurons was intact, consistent with a defect in sensory neuron activation. To explore sensory neuron activation we developed an in vivo preparation permitting the precise placement of a combined electrical and tactile stimulating probe upon eGFP-positive peripheral neurites. In wild-type larva electrical and tactile stimulation of peripheral neurites produced action potentials detectable within the cell body. In a subset of these sensory neurons an underlying generator potential could be observed in response to subthreshold tactile stimuli. A closer examination revealed that the amplitude of the generator potential was proportional to the stimulus amplitude. When assayed touché mutant sensory neurons also responded to electrical stimulation of peripheral neurites similar to wild-type larvae, however tactile stimulation of these neurites failed to uncover a subset of sensory neurons possessing generator potentials. These findings suggest that touché is required for generator potentials, and that cutaneous mechanoreceptors with generator potentials are necessary for responsiveness to light touch in zebrafish.
\end{abstract}

\section{Introduction}

The identification of mechanosensitive proteins in vertebrates using traditional approaches, such as affinity purification and expression cloning, has been hampered by the lack of highaffinity ligands and the likelihood that the mechanosensitive proteins must be coexpressed with additional membrane and cytoskeletal elements to function properly. Thus many researchers have looked to organisms amenable to forward genetic

\footnotetext{
Received March 29, 2010; revised May 4, 2010; accepted May 24, 2010.

The work reported here was supported by a grant from the National Science and Engineering Research Council of Canada (to L.S.-A.), a Canadian Institutes of Health Research operating grant (L.S.-A.), a Chercheur Boursier Award, and the Groupe de Recherche sur le Systéme Nerveux (entral from the Fond de Recherche en Santé du Québec (to L.S.-A.); National Institute of Neurological Disorders and Stroke Grant NS054731 (to J.Y.K.) and National Science Foundation Grant 0725976 (to J.Y.K.); a Long-Term Fellowship from the Human Frontier Science Program (to H.H.); and in part by Center for Organogenesis Training Grant 5-T32-HD007505 (to W.W.C.). We thank Pierre Drapeau, Mathieu Lachance, and members of the Kuwada and Drapeau laboratories for helpful comments regarding the preparation of this manuscript. We thank Guy Laliberté and Marina Drits for fish care. We also thank Dr. Hitohsi Okamoto (RIKEN, Wako-city Saitama, Japan) for the ssx-mini-ICP:eGFP stable transgenic line of zebrafish, Dr. Florian Engert (Harvard University, Boston, MA) for the ChR2-eYFP construct, and Dr. Angeles B. Ribera (University of Colorado, Denver, (0) for the mutant macho $\left(\right.$ ma $^{t t 261}$ ).

Correspondence should be addressed to Louis Saint-Amant, Université de Montréal, Roger-Gaudry Pavilion, S-546, 2900 Boulevard Édouard-Monpetit, Montréal, QC H3T 1J4, Canada. E-mail: louis.st-amant@umontreal.ca. H. Hirata's present address: Division of Biological Science, Nagoya University, Nagoya 464-8602, Japan.

W. W. Cui's present address: Department of Anesthesia and Perioperative Care, University of California, San Francisco, CA 94143

W. Zhou's present address: Life Science Institute, University of Michigan, Ann Arbor, MI 48109.

DOI:10.1523/JNEUROSCI.1639-10.2010

Copyright $\odot 2010$ the authors $\quad 0270-6474 / 10 / 309359-09 \$ 15.00 / 0$
}

screens, such as Caenorhabditis elegans and Drosophila, in the hopes of identifying mechanosensitive proteins conserved across evolution. However many of the candidate proteins identified from screens in these invertebrate organisms (Colbert and Bargmann, 1995; Walker et al., 2000; Liedtke et al., 2003; O'Hagan et al., 2005; Kindt et al., 2007) have been shown to be unessential for light touch in vertebrates (Sidi et al., 2003; Suzuki et al., 2003; Drew et al., 2004; Bautista et al., 2006; Kwan et al., 2006). These results raise the possibility that the proteins that mediate mechanotransduction in touch-sensitive neurons may not be conserved across phylogeny. Therefore we and others have turned to zebrafish (Granato et al., 1996; Haffter et al., 1996), a vertebrate model organism amenable to both genetic and in vivo electrophysiological manipulations.

Zebrafish embryos develop externally, possess a relatively simple nervous system, and respond to tactile stimuli within the first day of development (Saint-Amant and Drapeau, 1998). The neurons that activate touch-evoked behaviors are segregated into two groups: trigeminal ganglia neurons relay tactile stimuli delivered to the craniofacial region (Sneddon, 2003), and RohonBeard (RB) neurons within the spinal cord that relay tactile stimuli to the trunk and tail regions (Clarke et al., 1984). Both groups of neurons are likely polymodal as they have been shown to exhibit differential expression of several receptors implicated in nociception (Cockayne et al., 2000, 2005; Kucenas et al., 2003), and ubiquitous expression of TRPA1b which is essential for re- 
sponsiveness to the noxious compound mustard oil (Prober et al., 2008).

In a previous study, the membrane properties of RB neurons from several potential mechanosensitive mutants were chosen for detailed electrophysiological analysis (Ribera and Nüsslein-Volhard, 1998). Results from this study revealed that most mutant sensory neurons possessed defects within their excitable properties, and therefore are not strong candidates for mutations affecting mechanosensation. Recognizing the need for additional touch-unresponsive mutants we undertook another forward genetic screen to identify novel mutants with abnormal touch-evoked behaviors. From our screen one family (mi173), subsequently named touché (tou ${ }^{\text {miliz3 }}$ ), was found to harbor a recessive mutation which resulted in offspring that were selectively unresponsive to light touch. Employing a novel in vivo recording technique we failed to uncover a subset of sensory neurons in touché mutants which responded to tactile stimulation with generator potentials. These findings indicate that touché is required for generator potentials, and that sensory neurons with generator potentials underlie responsiveness to light touch in zebrafish.

\section{Materials and Methods}

Animal care and use. Zebrafish were bred and raised according to approved guidelines set forth by the Animal Experimentation Ethics Committee, Université de Montréal and the University Committee on Use and Care of Animals, University of Michigan. Staging of embryos was performed as described previously (Kimmel et al., 1995). The touché allele mi173 $\left(\right.$ tou $\left.^{\text {mil73 }}\right)$ was identified in a screen conducted at the University of Michigan, Ann Arbor following previously published procedures (Haffter and Nüsslein-Volhard, 1996).

Mapping. A mapping family for touché was established by crossing a touché male carrier (Michigan genetic background) with a wild-type WIK female (Zebrafish Resource Center, Eugene, Oregon). Offspring from this mapping family were subjected to bulk segregate analysis (Postlethwait et al., 1994) according to the Zon laboratory protocol (http://zfrhmaps.tch.harvard.edu) using 20 wild-type sibling and 20 touché mutants.

Behavioral analysis. All reagents were obtained from Sigma-Aldrich unless otherwise noted. Embryos at $\sim 24$ h postfertilization (hpf) were dechorionated with pronase, staged and segregated according to their responsiveness to touch. Tactile and nociceptive stimuli were delivered by striking the tail of an embryo with a sideways motion up to 3 times with a pair of No.5 forceps and pinching of the tail, respectively. The speed of tactile stimuli was assessed offline by measuring tip displacement (Fig. $1 A$ ) as a function of time. Embryos were exposed to noxious stimuli individually in 24 well plates: allyl-isothiocyanate (Acros Organics) at $500 \mu \mathrm{M}$ mustard oil in sham (1\% DMSO, v/v in Evans solution), or acidified Evans solution ( $\mathrm{pH} 4.5$ adjusted with acetic acid, sham Evans, $\mathrm{pH} 7.5)$.

Electrically evoked bouts of swimming used in the stopping assay were elicited using a bipolar electrode and an A-M Systems Model 2100 isolated pulse stimulator in larvae embedded in $1 \%(\mathrm{w} / \mathrm{v})$ low-melting point agarose. To mimic head-first collisions a jet of water was applied to the head (20 psi, $50 \mathrm{~ms}$ ) using a Picospritzer III (Parker Hannifin). The electrical stimulus was paired with an LED (see Fig. $3 A$ ), and the timing of both was controlled by pClamp 8 software using a Digidata 1200 interface (Molecular Devices).

Acoustic-vestibular responses were assayed between 2 and $4 \mathrm{~d}$ postfertilization (dpf). At $2 \mathrm{dpf}$ the ability of lateral-line stimuli to activate motor behaviors was examined by placing larvae individually into the center of a $60 \mathrm{~mm}$ dish in $3 \mathrm{ml}$ of fish water (Westerfield, 2000). To the side of the dish was added $1 \mathrm{ml}$ of fish water by pipette to cause passive displacement of the larvae. Larvae were scored as positive responders if motor activity was observed before the larvae stopped moving passively. The contribution of inner-ear hair cell function to body posture was assayed at $3 \mathrm{dpf}$ by counting the number of dorsal-up orientated larva. The ability to respond to acoustic stimuli was assayed by placing larvae into $60 \mathrm{~mm}$ dishes in $4 \mathrm{ml}$ of fish water atop a metal plate. The plate was sharply struck with a metal rod from a constant height to cause a loud audible sound. Some experiments were performed on the mutants and siblings simultaneously to rule out subtle changes in threshold for detection.

Light-evoked (480 $\mathrm{nm}$ ) activation of motor behaviors was performed by injecting $50 \mathrm{pg}$ of plasmid containing channelrhodopsin-2 coupled to enhanced yellow fluorescent protein (ChR2-eYFP) under the control of the Isl1-SS-enhancer:Gal4-VP16:UAS-E1b promoter (Douglass et al., 2008 ) diluted in $5 \mathrm{nl}$ of water containing $0.01 \%$ phenol red into freshly fertilized embryos from touché carrier incrosses at the 1-2 cell stage using a Nanoinject II system (Drummond Scientific). Embryos were screened at $\sim 24-27 \mathrm{hpf}$ for expression eYFP and touch responsiveness using an Olympus dissecting microscope (SZX7) fitted with epifluorescence. Light-evoked motor behaviors were triggered manually by opening a shutter on individual embryos in 24 well plates.

Behaviors were recorded at 30 and $200 \mathrm{~Hz}$ using Flea2 (FL2-20S4M-C) and Grasshopper (GRAS-03K2M-C) cameras from Point Gray (Richmond, BC). Images were captured with PRG Flycap, and analyzed offline using ImageJ (http://rsbweb.nih.gov/ij/).

FM1-43 dye uptake and immunohistochemistry. For dye uptake FM1-43 (Invitrogen), a dye thought to enter hair cells through the mechanotransduction channel, was applied at $3 \mu \mathrm{M}$ in Evans solution (see below) for $30 \mathrm{~s}$. Thereafter, larvae were washed in Evans solution containing the local anesthetic tricaine $(0.02 \% \mathrm{w} / \mathrm{v})$ five times for $10 \mathrm{~s}$ each. Dye uptake was assessed on a dissecting microscope fitted with a QICAM Fast 1394 camera from QImaging.

For labeling eGFP and ChR2-eYFP larvae were fixed in 4\% paraformaldehyde for $30 \mathrm{~min}$ and then washed five times for $10 \mathrm{~min}$ in washing 
A

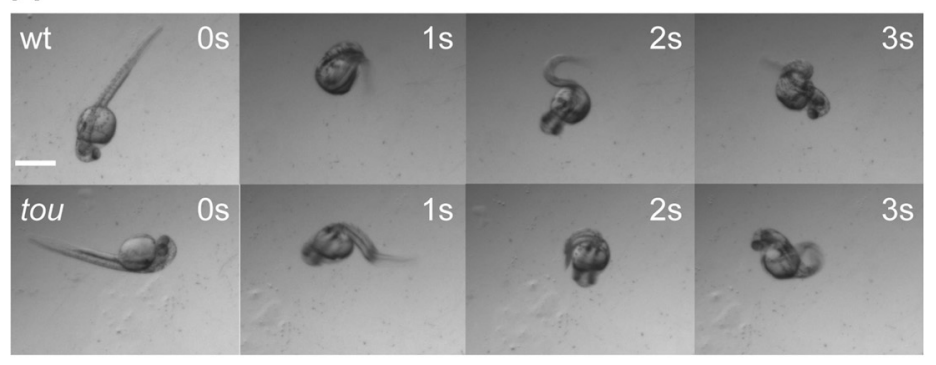

B

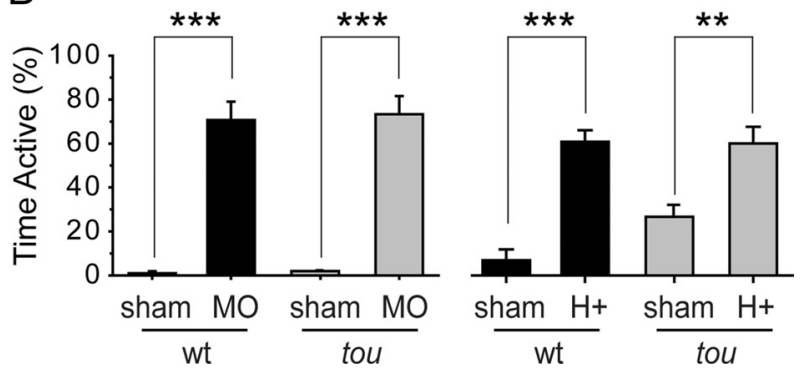

Figure 2. touché mutants respond normally to noxious stimuli. A, Time-lapse images of wild-type and touché mutant motor behaviors in the presence of mustard oil (500 $\mu \mathrm{M}$ ) at $26 \mathrm{hpf}$. Scale bar, $500 \mu \mathrm{m}$. $B$, Analysis of percentage time active within the first $10 \mathrm{~s}$ of exposure to mustard oil (MO; $n=28$ embryos from 3 clutches), and within $10 \mathrm{~s}$ following $1 \mathrm{~min}$ of exposure to low pH (H+; $n=15$ embryos from 3 clutches; images not shown) compared with sham controls for mustard oil (1\% DMSO) and low pH (Evans solution, pH 7.5), respectively. ${ }^{* *} p \leq 0.01,{ }^{* * *} p \leq 0.001$.

A

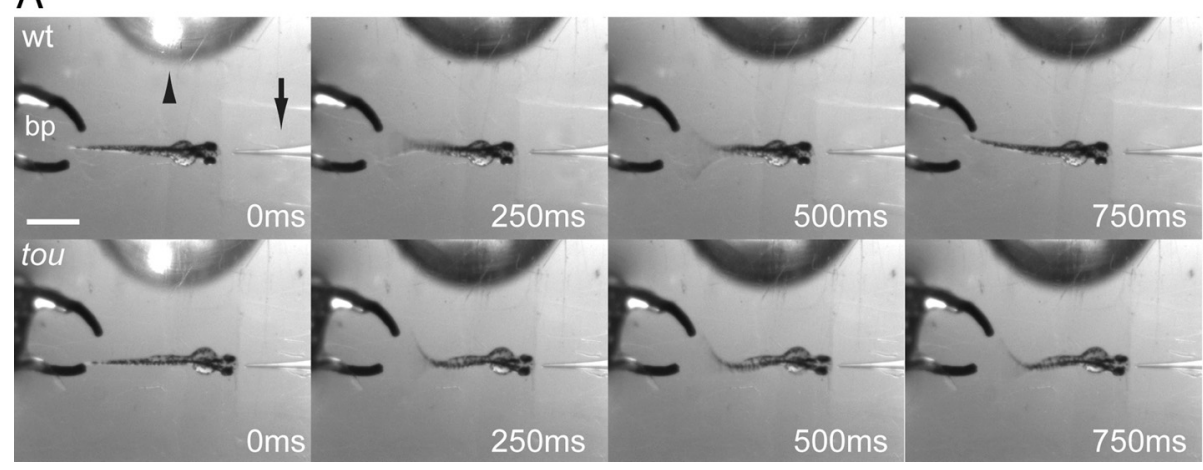

B

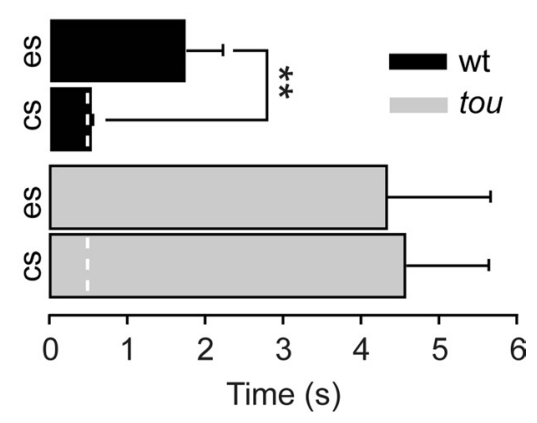

Figure 3. touché mutants lack a stopping response. $A$, Time-lapse images of wild-type and touché mutants at $48-52 \mathrm{hpf}$ during electrically evoked swimming trials wherein the counterstimulus was applied. The electrical stimulus delivered by a bipolar probe (bp) was paired with an LED (arrowhead) visible within the field of view. The counterstimulus, waterjet (arrow), was applied $500 \mathrm{~ms}$ following the electrical stimulus. Scale bar, $500 \mu \mathrm{m}$. B, Time spent swimming following the electrical stimulus (es) alone, or in trials where the counterstimulus (cs) was applied. The counterstimulus is indicated as a dashed line ( $n=10$ larvae for each from 3 clutches). ${ }^{* *} p \leq 0.01$.

buffer (PBS containing $0.1 \%$ Triton X-100), and two times for $10 \mathrm{~min}$ in blocking buffer (wash buffer containing $2 \mathrm{mg} / \mathrm{ml}$ bovine serum albumin). Rabbit anti-EGFP (Torrey Pines Biolabs) primary was diluted $1 / 1000$ in blocking buffer and bound overnight. Thereafter larvae were washed five times for $10 \mathrm{~min}$ in washing buffer, two times for $10 \mathrm{~min}$ in blocking buffer. Secondary antibody (anti-rabbit Alexa-488, Invitrogen) diluted 1/1000 in blocking buffer was bound for $4 \mathrm{~h}$. Larvae were then washed five times for $10 \mathrm{~min}$ with washing buffer and then mounted in $70 \%$ glycerol in PBS. Images were captured with a spinning disk confocal (Quorum) microscope (Olympus, BX-51). Three segments above the anus were analyzed for RB cell counts. For trigeminal neuron cell counts the average diameter of a trigeminal neuron was first determined to be $\sim 8 \mu \mathrm{m}$. Optical stacks of trigeminal ganglia were then divided into $8 \mu \mathrm{m}$ optical sections, starting $4 \mu \mathrm{m}$ into the ganglion, and neurons with discernable cell bodies were counted under double blind conditions. Innervation of the skin by peripheral neurites was determined by thresholding all neurite projections in the skin for the 3 somites centered on the anal somite under double blind conditions. Thereafter the percentage of the total thresholded area relative to total area was determined using ImageJ.

Recording methods. Electrophysiological recordings from zebrafish were obtained from neurons at room temperature using methods similar to those previously described (Hamill et al., 1981; Ribera and NüssleinVolhard, 1998; Drapeau et al., 1999). In brief larvae were anesthetized in Evans recording solution (in $\mathrm{mm}$ ): $134 \mathrm{NaCl}, 2.9 \mathrm{KCl}, 2.1 \mathrm{CaCl}_{2}, 1.2$ $\mathrm{MgCl}_{2}, 10$ glucose, 10 HEPES, pH 7.5 with $\mathrm{NaOH}$ containing $0.02 \%$ $(\mathrm{w} / \mathrm{v})$ tricaine. The skin of a larva pinned to a $35 \mathrm{~mm}$ Sylgard-coated dish was removed with a pair of No.5 forceps. The solution was exchanged with Evans solution containing $15 \mu \mathrm{M}$ curare flowing throughout the recording session at $\sim 1 \mathrm{ml} / \mathrm{min}$. To gain access to the spinal cord the bath solution was replaced with recording solution containing $4 \mathrm{mg} / \mathrm{ml}$ collagenase type XI and incubated until the muscle started to separate $(\sim 10$ $\min$ ). Thereafter the muscle was peeled away using suction applied to a broken pipette $(\sim 50 \mu \mathrm{m})$. The internal recording solution contained the following (in $\mathrm{mm}$ ): $116 \mathrm{~K}$-gluconate, $16 \mathrm{KCl}, 2 \mathrm{MgCl}_{2}, 10 \mathrm{HEPES}, 10$ EGTA, at pH 7.2 with $\mathrm{KOH}$ and $0.1 \%$ SulforhodamineB for cell type identification. Borosilicate glass electrodes had resistances of 5-8 $\mathrm{M} \Omega$ when filled with internal recording solution. Recordings were made with an Axopatch 200B amplifier (Molecular Devices) low-passed filtered at $1-5 \mathrm{kHz}$ and sampled at $1-10 \mathrm{kHz}$. Electrical and tactile stimuli were delivered through the use of a bipolar stimulating probe (A-M Systems Model 2100) controlled by a piezoelectric stimulator (Moffatt and Hume, 2007). Probes were placed upon eGFP-positive neurites $\sim 100$ $\mu \mathrm{m}$ from the cell body. Generator potential amplitudes were normalized for $\mathrm{RB}$ cell input resistance, and scaled to zero which represents the displacement preceding the first observable membrane depolarization as described previously (Drew et al., 2002). Data acquisition was controlled by pClamp 10 software using a Digidata 1440A interface. The initial data analysis was done with Clampfit 10, and figures were prepared using SigmaPlot 11.0.

\section{Results}

\section{touché is a novel light touch-unresponsive mutant}

In contrast to other organisms a clear difference between light touch and nociceptive stimuli, and their related behavioral responses, has not been defined for zebrafish. In an attempt to address this deficit we first examined the behavioral responsiveness of zebrafish embryos to increasing forces delivered by calibrated animal hairs and commercially available von Frey filaments. However the lowest hairs and filaments obtainable ( 5-8 mg) consistently resulted in the activation of motor behaviors in zebrafish embryos. As work from other organisms predicted that zebrafish embryos might display a graded responsiveness to increasing forces we reasoned that $\sim 5 \mathrm{mg}$ was greater 
than the minimal force detectable by zebrafish embryos. Therefore we explored whether varying the speed at which a tactile stimulus was delivered might uncover speeds, and related forces at which zebrafish embryos exhibited a graded responsiveness to tactile stimuli. In response to stimuli below $5 \mathrm{~mm} / \mathrm{s}$ all zebrafish embryos failed to respond to touch (Fig. $1 A, B$ ). Increasing the speed of delivery resulted in a corresponding increase in the percentage of responsive embryos, until at $20 \mathrm{~mm} / \mathrm{s}$ when all embryos examined were responsive. Closer examination also revealed that the maximum number of coils performed by zebrafish embryos in response to light touch was three.

To elicit a more nociceptive response the tips from a pair of forceps were closed upon the caudal fin, herein referred to as tail pinching (Fig. $1 C$ ). In response to tail pinching, zebrafish embryos were found to consistently perform 3 or more coils, with a range of $3-12$ coils (Fig. $1 D$ ). Thus zebrafish embryos exhibit at least two different levels of responses to increasing forces, with light touch stimuli evoking $\leq 3$ coils and nociceptive stimuli evoking $\geq 3$ coils.

In a screen for novel zebrafish mutants exhibiting abnormal touch-evoked motor behaviors one family (mi173), subsequently named touché (tou ${ }^{\text {mil73 }}$ ), was found to harbor a recessive mutation which resulted in offspring that were unresponsive to light touch (Fig. 1A,B), but retained the ability to respond to tail-pinching (Fig. $1 C, D)$. To determine whether touché represented a new touch-unresponsive mutant we rough mapped the touché locus to chromosome 2. Of the previously identified touch-unresponsive mutants (Granato et al., 1996) only macho ( mao $^{t t 261}$ ) has been mapped to chromosome 2 (www.zfin. org), and therefore complementation analysis was performed with macho. Pairwise crosses between macho and touché complemented the mutant phenotypes indicating that touché is not a new allele of macho. Collectively these findings indicate that touché represents a new touchunresponsive mutant, deficient in the ability to respond light touch.

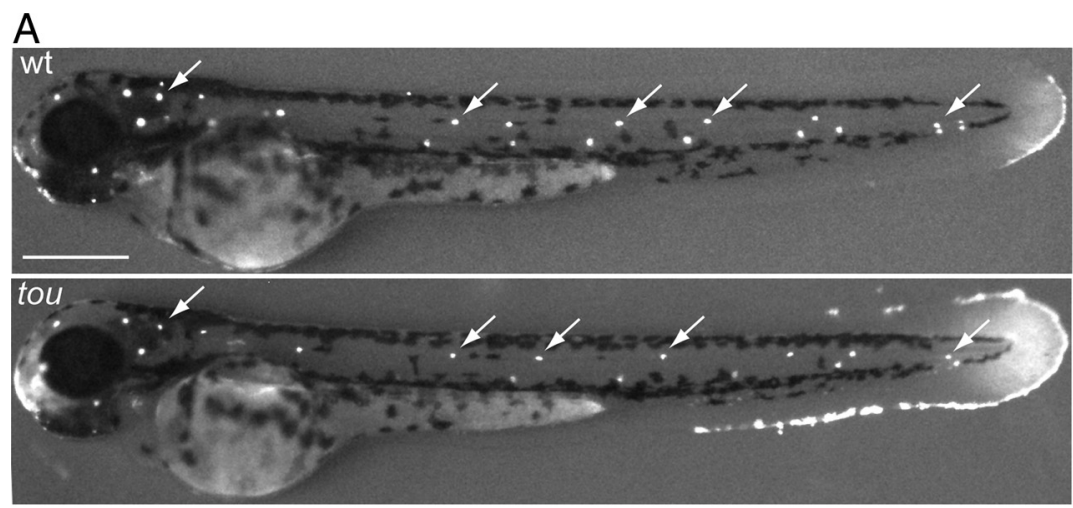

B
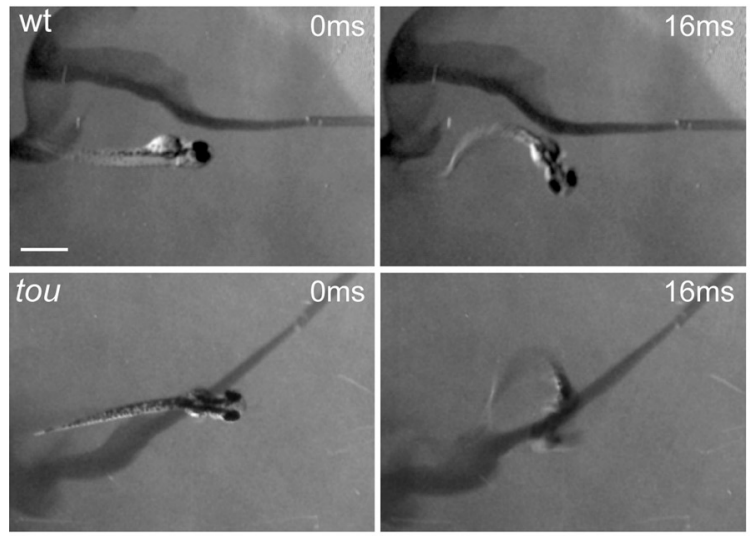

C

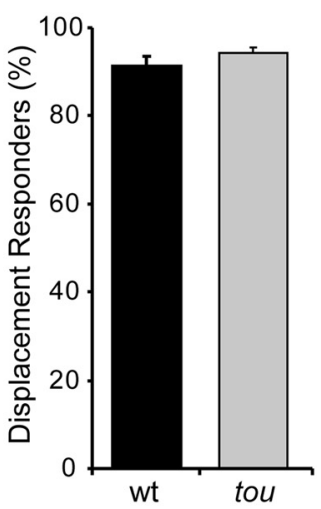

D
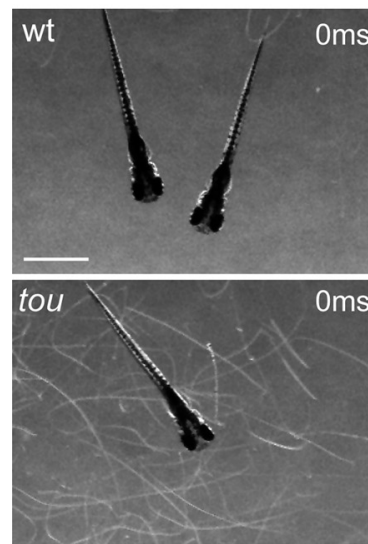

Oms

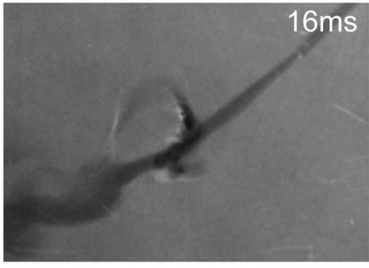

E
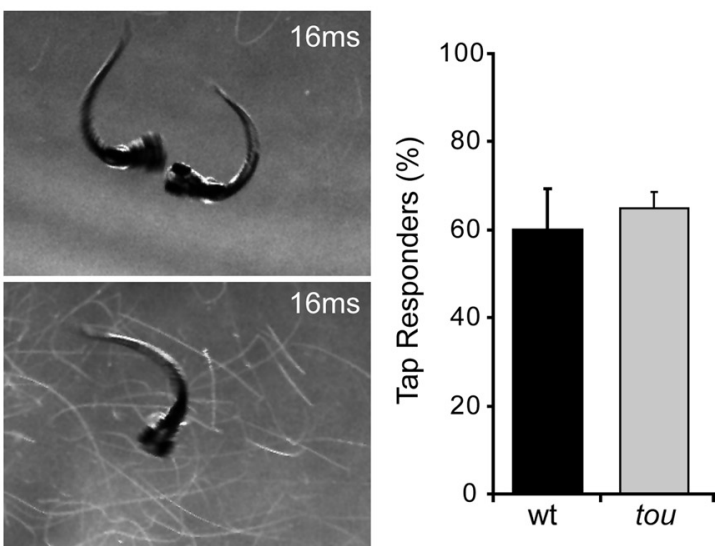

Figure 4. touché mutants respond to acoustic-vestibular stimuli. $A$, FM1-43 dye uptake by hair cells of the lateral-line (arrows indicate the location of hair cells within a few neuromasts) is present in both wild-type and touché mutants $48-52$ hpf. 0 f note black regions are melanophores, and whitening along the caudal fin is background dye labeling. Scale bar, $200 \mu \mathrm{m}$. $\boldsymbol{B}$, Time-lapse images of wild-type and touché mutant larvae $48-52 \mathrm{hpf}$. A lateral line stimulus (rapid water flow, dark stream) was delivered along the body axis. Scale bar, $500 \mu \mathrm{m}$. C, Responsiveness of wild-type ( $n=99$ from 4 clutches) and touché mutants to lateral-line stimuli ( $n=92$ from 4 clutches). D, Time-lapse images of responses of wild-type and touché mutant larvae $96-100 \mathrm{hpf}$ to tapping of the dish. Scale bar, $1 \mathrm{~mm}$. $E$, Responsiveness of wild-type and touché mutants to dish tapping ( $n=80$ from 4 clutches for each). touché mutants are responsive to multiple noxious stimuli

The finding that touché mutants were unresponsive to light touch, but responded to tail-pinching prompted an investigation into the responsiveness of touché mutants to other modes of sensory stimuli. During the first few days of development, zebrafish are responsive to the compound commonly known as mustard oil (allyl-isothiocyanate) and to low $\mathrm{pH}$. The behavioral responsiveness of zebrafish to mustard oil was shown to require TRPA1b, a member of the TRP superfamily of cation channels expressed by zebrafish sensory neurons (Prober et al., 2008). To examine the responsiveness of wild-type and touché mutants to mustard oil, embryos were transferred into Petri dishes preloaded with mustard oil. Under control conditions (Evans solution containing 1\% DMSO) wild-type and touché mutants were found to be predominantly inactive (Fig. 2A,B). However when exposed to mustard oil wild-type and touché mutants were both significantly more active. Comparisons failed to uncover a significant difference in the time active in mustard oil between wildtype and touché mutants. 
A

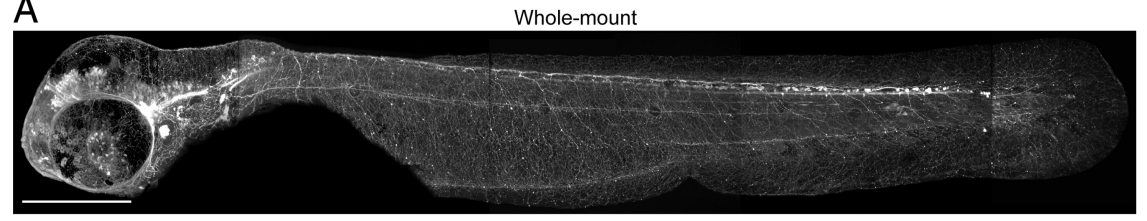

B
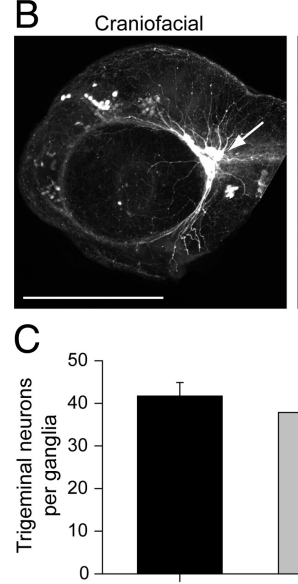

wt

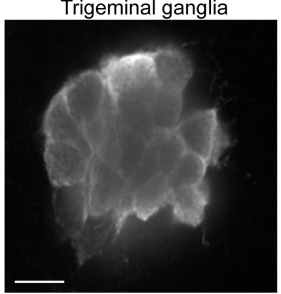

Peripheral neurites
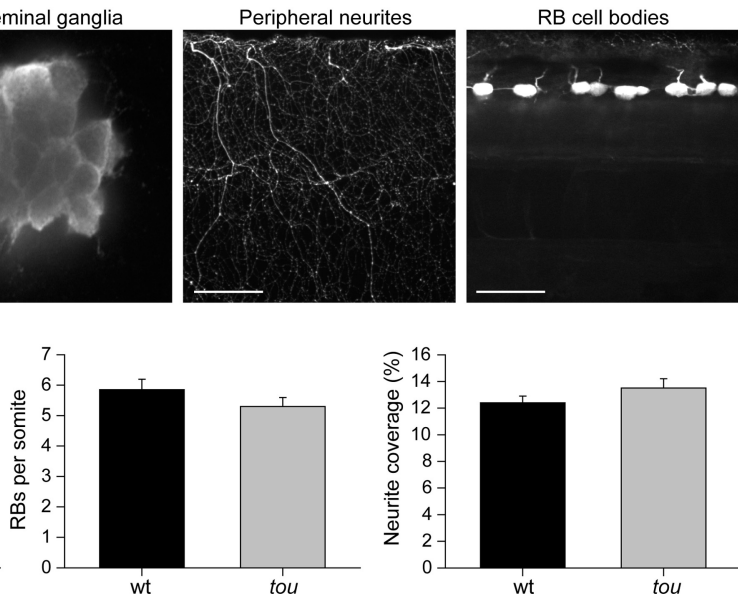

wt

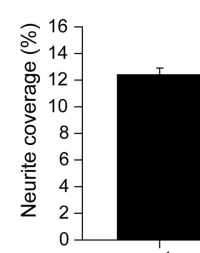

wt

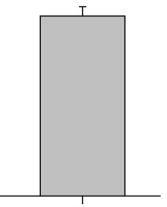

tou

Figure 5. Gross morphology of sensory neurons in touché mutants appears normal. $A$, Whole-mount labeling of eGFP-positive sensory neurons from a touché mutant larvae at $52 \mathrm{hpf}$; scale bar, $200 \mu \mathrm{m}$. B. Craniofacial labeling with trigeminal ganglia highlighted (arrow); scale bar, $200 \mu \mathrm{m}$. Trigeminal ganglia; scale bar, $10 \mu \mathrm{m}$. Peripheral neurites; scale bar, $50 \mu \mathrm{m}$. RB cell bodies; scale bar, $50 \mu \mathrm{m}$. C, Average trigeminal neurons per ganglia, RBs per somite, and coverage of skin by peripheral neurites ( $n=9$ for each, from 3 clutches).

In contrast to mustard oil, the process by which low $\mathrm{pH}$ is perceived by zebrafish and transformed into a behavioral response is unknown. However it is likely to involve member(s) of the acid sensing ion channel (ASIC) family and/or TRPV1, which are known to be expressed by a subset of sensory neurons in zebrafish (Paukert et al., 2004; Caron et al., 2008). To determine whether touché mutants were capable of perceiving and responding to low $\mathrm{pH}$ we exposed larvae to acidified Evans recording solution, $\mathrm{pH}$ 4.5. When compared to normal Evans ( $\mathrm{pH} 7.5$ ), wild-type and touché mutants were both found to be significantly more active (Fig. 2 B). Comparisons of time active between wildtype and touché mutants again failed to uncover a significant difference. Thus touché is not required for the behavioral responsiveness to these noxious stimuli.

\section{touché mutants lack a stopping response}

After the onset of swimming it was noted that touché mutants often failed to stop swimming upon head-first collisions with the sides of dishes. This "stopping response" has been shown to require feedback from mechanosensitive trigeminal neurons in $\mathrm{Xe}$ nopus laevis embryos (Boothby and Roberts, 1992a,b). To examine this finding in detail, larvae were restrained in agar, with the tail and head regions exposed to allow for the delivery of stimuli and swimming. Larvae were then induced to swim by passing a current across the caudal tail using a bipolar stimulating electrode (Fig. 3A), which has been shown to activate zebrafish sensory neurons in vivo (Higashijima et al., 2003). In an equal number of trials a puff of water referred to as the counterstimulus was delivered to the head $500 \mathrm{~ms}$ after an electrical stimulus. In wild-type larvae the counterstimulus resulted in a significant reduction in the duration of swimming (Fig. $3 B$ ). In contrast, no significant reduction in the duration of swimming was observed in touché mutants following the counterstimulus. Therefore touché mutants lack two sensory-evoked behaviors known to require input from mechanosensitive neurons.

touché mutants respond to acoustic-vestibular stimuli

To rule out a wholesale disruption of mechanosensitive process in touché mutants, we assayed behaviors dependent upon input from mechanosensitive hair cells of the inner ear and the lateral-line. Mechanotransduction within these hair cells allow zebrafish to respond to water displacement, acoustic stimuli (tapping), and contribute to a dorsal-up body posture. As a first level of characterization of mechanotransduction within these hair cells, we exposed embryos to the dye FM143, which is thought to enter hair cells through the mechanotransduction channel (Seiler and Nicolson, 1999). Following incubation in FM1-43, wild-type and touché mutant hair cells both exhibited uptake of the fluorescent dye (Fig. 4A).

Behavioral defects mediated by mutations in proteins essential to the function of the lateral-line and inner ear hair cells become obvious between the second and fourth days of development (Nicolson et al., 1998). When assayed, no differences in the responsiveness to water displacement (Fig. $4 B, C$ ), or tapping (Fig. $4 D, E$ ) were found between wild-type and touché mutants. Similarly wild-type and touché mutants were both found to be dorsally orientated on day 4 (Fig. $4 B, D)$. Thus mechanotransduction within touché mutant hair cells appears present, indicating that the touché mutation does not affect all mechanically sensitive cells.

\section{Sensory neuron anatomy in touché mutants}

The behavioral results thus far suggest a role for touché within touch-sensitive neurons. This role could be functional, such as in the relay of tactile stimuli to second order neurons. Alternatively touché could be required developmentally for the differentiation and/or retention of touch-sensitive neurons. As a first step in distinguishing between these two possibilities, the presence and morphology of sensory neurons in zebrafish were compared between wild-type and touché mutants. In zebrafish, tactile stimuli are conferred by two groups of touch-sensitive neurons: trigeminal neurons relay touch to the craniofacial region and RB cells relay touch to the trunk and tail regions. To facilitate the identification and characterization of trigeminal neurons and RB cells, the touche mutation was crossed into a stable transgenic line (Uemura et al., 2005) expressing eGFP under the control of a sensory neuron enhancer-promoter (ssx-mini-ICP:eGFP). When compared with wild-type siblings, touché mutants were found to possess a similar number of RB cells per somite, trigeminal neurons per ganglia, and an indistinguishable amount of neurite coverage by sensory neurons (Fig. 5). Thus there does not appear to be a gross morphological difference between the sensory neurons of wildtype and touché mutants.

\section{Light-evoked activation of sensory neurons triggers motor behaviors in touché mutants}

Given that sensory neurons appear normal in touché mutants we examined whether the exogenous activation of sensory neurons could trigger motor behaviors. Recently, exposure to blue light in embryos expressing ChR2-eYFP under the control of a sensory 
neuron promoter was shown to be sufficient to trigger action potentials within trigeminal neurons, and activation of motor behaviors (Douglass et al., 2008). The injection of the ChR2-eYFP construct resulted in embryos with several fluorescent cells in locations consistent with sensory neurons (Fig. 6A). We found that exposure to blue light $(\sim 1 \mathrm{~s})$ at $\sim 27 \mathrm{hpf}$ triggered motor behaviors in both wild-type and touché mutant embryos positive for eYFP expression (Fig. $6 B$ ). In contrast, exposure to red light failed to evoke motor behaviors in either wild-type or touché mutant embryos positive for eYFP expression (Fig. 6C). As a control, embryos expressing eGFP within the same neurons failed to respond to blue light exposure. Thus the reaction to blue light required the injection of the ChR2-eYFP construct. These findings indicate that transmission of electrical signals downstream of action potential generation in sensory neurons appears present, and capable of activating motor behaviors within touché mutants.

\section{Excitable properties of touché mutant sensory neurons are normal}

Previously the development of ionic currents in zebrafish RB neurons were examined (Ribera and Nüsslein-Volhard, 1998), wherein the maturation of an overshooting action potential in sensory neurons was suggested to underlie the development of touch responsiveness. This hypothesis was supported by the examination of sensory neuron membrane properties from the touch-unresponsive mutant macho, which revealed that macho mutants fail to develop overshooting action potentials despite the normal development of other ionic currents. Based on these findings the excitable properties of touché RB neurons were examined in vivo using a preparation wherein the skin and muscle contralateral to target $\mathrm{RB}$ neurons was removed leaving the peripheral neurites intact (Fig. 7A). Using this preparation we found that $\mathrm{RB}$ neurons from both wild-type and touché mutants initiate action potentials in response to depolarizing current injections to the cell body (Fig. 7B). When compared wild-type and touché mutant RB neurons were found to exhibit similar resting membrane potentials, action potential thresholds, and amplitudes of overshoot and undershoot (Fig. 7C).

Next a bipolar stimulating probe, attached to a piezoelectric motor, was placed upon eGFP-positive neurites $\sim 100 \mu \mathrm{m}$ from the cell body in the stable transgenic line described above (Fig. 5). This approach allowed for the electrical and mechanical stimulation (see below) of the same neurite belonging to one RB neuron. In both wild-type and touché mutant RB neurons, depolarization of the peripheral neurite evoked action potentials detectable within the cell body (Fig. 7B). A similar stimulus applied $\sim 15 \mu \mathrm{m}$ rostral or caudal to the peripheral neurite failed to activate sensory neurons indicating that the bipolar stimulating probe was focally exciting neurites. When again compared wild-type and touché mutant RB neurons were found to exhibit similar action potential thresholds, and generated action potentials with similar amplitudes of overshoot and undershoot (Fig. 7C). Thus the lack of responsiveness to light touch in touché mutants cannot be explained by a difference in the excitability of touché mutant sensory neurons, or by a breakdown in electrical signals from the peripheral neurites to the cell body.

\section{touché mutants lack sensory neurons with generator potentials}

To examine whether tactile stimuli delivered to the peripheral neurite could evoke action potentials detectable within the cell body in touché mutants the bipolar stimulating probe was driven into the peripheral neurite with a piezoelectric motor. Previously the movement of probes with the piezoelectric motor was shown to be controllable on the $\mu \mathrm{m}$ level, with movements being completed within hundreds of microseconds (Moffatt and Hume, 2007). We found that in wild-type RB cells, wherein electrical stimulation of the peripheral neurite-evoked action potentials detectable within the cell body (Fig. 7B), mechanical stimulation 
A

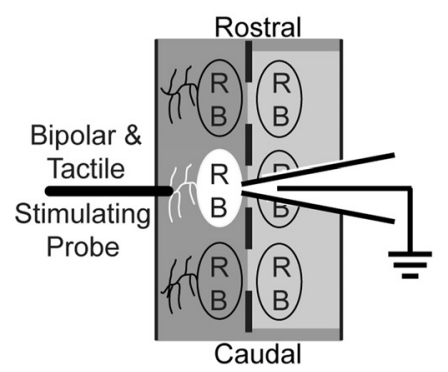

B

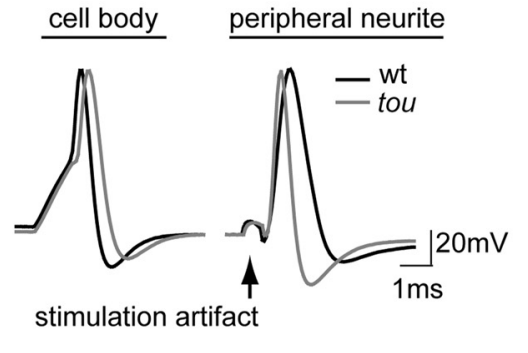

C

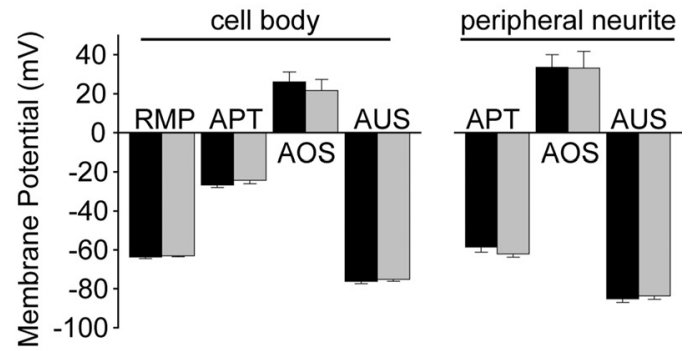

Figure 7. Electrical stimulation of peripheral neurites triggers action potentials within touché mutant RBs. $A$, Schematic of the in vivo recording preparation, which allows for electrical and mechanical stimulation of the same peripheral neurite. Dashed line indicates dorsal midline. Light gray area indicates the region in which the skin and muscle were removed to allow access to a contralateral RB cell body with an intact peripheral neurite (white). B, Depolarizing intracellular current injections ( 2 ms) to cell bodies (left panel) and extracellular stimulation (1 ms) of peripheral neurites (right panel). C, Analysis of resting membrane potential (RMP), action potential thresholds (APT), amplitude of overshoots (A0S), amplitude of undershoots (AUS) in response to depolarizing current stimulation of cell bodies and peripheral neurites for both wild-type $(n=19)$ and touché mutants $(n=16)$.

also triggered action potentials detectable within the cell body $(n=19 / 19$; Fig. 8 A). A closer examination revealed that $\mathrm{RB}$ cells in wild-type larvae could be subdivided into two groups: those possessing generator potentials (type I, $n=9 / 19$ ), and those wherein a generator potential was not observed (type II, $n=$ 10/19; Fig. 8A). Employing the same stimulating technique in touché mutants, which had also responded to electrical stimulation of the peripheral neurite $(n=16 / 16)$, failed to uncover RB cells with generator potentials (type I, $n=0 / 16$ ).

Generator potentials are graded membrane depolarizations induced in the termini of touch-sensitive neurons, which upon reaching sufficient amplitudes, trigger action potentials in sensory afferents. To examine whether the membrane depolarizations observed in zebrafish RB neurons in response to mechanical stimuli were generator potentials a series of subthreshold mechanical stimuli were delivered to the peripheral neurite of several RB neurons $(n=6)$. In response to increasing mechanical stimuli membrane depolarizations were found to be graded (Fig. $8 B$ ), and after normalizing for RB neuron input resistances (Fig. $8 C)$ strongly correlated with displacement $\left(r^{2}=0.97\right)$. Finally to ensure that the graded membrane depolarizations were not failed action potentials we applied tetrodotoxin to the recording chamber which prevented spiking $(n=2)$, but failed to block the graded membrane depolarizations indicating that the observed graded membrane depolarizations were in fact generator potentials. Collectively these findings suggest that the touché mutant phenotype results from a lack of sensory neurons with generator potentials, and implies that light touch in zebrafish is conveyed by mechanosensitive neurons with generator potentials.

\section{Discussion}

In a screen for novel touch-unresponsive zebrafish mutants we uncovered one mutant, subsequently name touché, which was unresponsive to light touch. Although rough mapping indicated that touché could be a new allele of macho, a previously identified touch-unresponsive mutant (Granato et al., 1996), pairwise crosses with macho carriers complemented the touché mutant phenotype. Thus touché represents a new touch-unresponsive mutant.

Upon closer examination of the touché mutant phenotype it was noted that touché mutants also lacked a stopping response, a behavior known to require input from trigeminal touch-sensitive neurons (Boothby and Roberts, 1992a,b). This finding suggested that the touché mutation might affect all mechanosensitive processes, however touché mutants were found to exhibit acoustic- vestibular behaviors which rely on input from mechanosensitive hair cells. Coupled with the normal responsiveness of touché mutants to noxious stimuli (mustard oil and low $\mathrm{pH}$ ) implies that the touché mutation affects behaviors dependent upon input from touch-sensitive neurons.

Potentially in support of a requirement for touché in the regulation of behaviors dependent upon input from touch-sensitive neurons were the findings that touché mutants swam for longer durations following electrical stimulation, and were more spontaneously active at $4 \mathrm{~d}$, a time point wherein larvae are rather immotile and often found to be stuck to the sides of objects. These findings may reflect an absence of sensory feedback from mechanosensitive neurons such as those that innervate mucous cells which likely alert zebrafish larvae that they have encountered an object suitable for adherence similar to Xenopus laevis embryos (Boothby and Roberts, 1992a,b). Alternatively the increase in spontaneous activity in mutants could reflect the absence of an inhibitory influence of touché on the locomotor network which underlies swimming in zebrafish, or a requirement of sensory input in modulating locomotor network activity.

In an attempt to understand the contribution of touche to the activation of touch-evoked behaviors we first considered that the inability of touché mutants to respond to light touch could be the result of absent, or aberrant touch-sensitive neurons. However comparisons between wild-type and touché mutants revealed that mutants possessed a similar number of RB neurons per somite, trigeminal neurons, and extent of skin innervation by peripheral neurites. While these findings suggest that the mutant phenotype is the result of a functional loss of touché within touchsensitive neurons a limitation of this comparison exists which stems from a general lack of knowledge concerning sensory neurons in zebrafish. Presently it is unknown whether zebrafish sensory neurons are a heterogenous or homogenous population of mono or polymodal-sensitive neurons. Therefore while touché mutants have a similar overall number of sensory neurons, touché mutants could lack a subtype of sensory neuron responsive to light touch while having more of another type. A detailed analysis of sensory neuron responsiveness to various stimuli in zebrafish is needed to examine this possibility.

We next considered that touché might be required functionally within touch-sensitive neurons for the transduction of tactile stimuli to second order neurons. This requirement could be (but not limited to) within the mechanotransduction complex responsible for the transformation of tactile stimuli into an electri- 


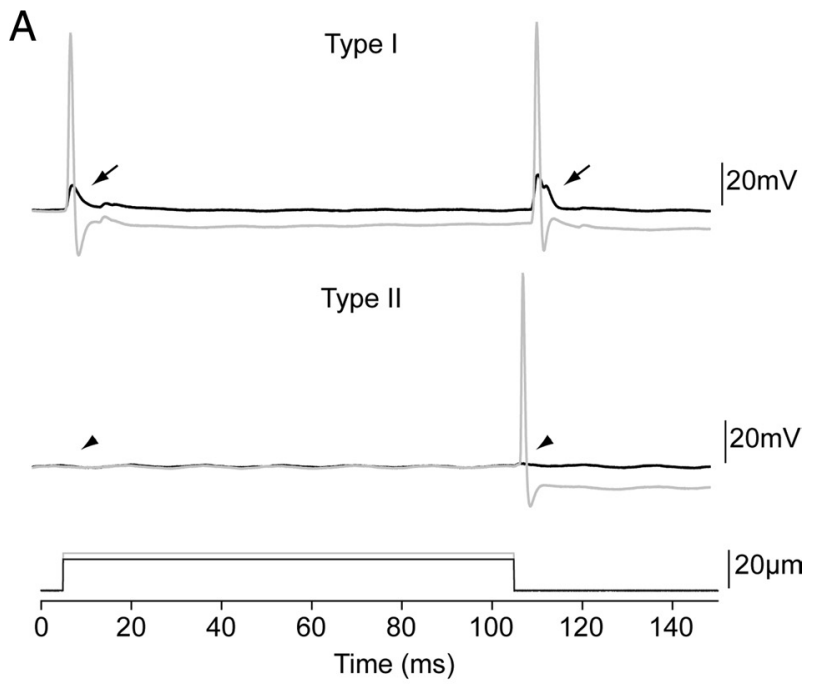

B
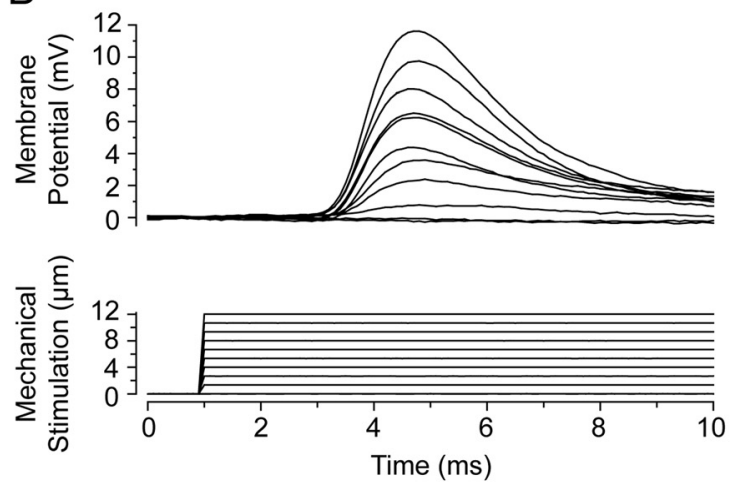

C

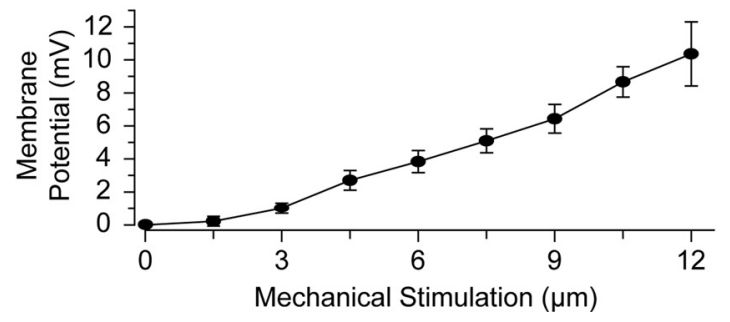

Figure 8. touché mutants lack sensory neurons with generator potentials. $\boldsymbol{A}$, Top, In a subset of RB cells (type I, $n=9 / 19$ ), a subthreshold mechanical stimulus (black) applied to the peripheral neurite results in a generator potential (arrows) at the onset and offset of the stimulus. In these RB cells, increasing the amplitude of stimuli (gray) triggers action potentials at the onset and the offset of the stimulus. Bottom, Other RB cells (type II; $n=9 / 19$ ) activated by mechanical stimuli (gray) lack generator potentials (arrowheads) in response to subthreshold stimuli (black). $\boldsymbol{B}$, Generator potentials activated in response to increasing mechanical stimuli. $\boldsymbol{C}$, The amplitude of generator potentials correlates with the amplitude of mechanical stimuli $(n=6)$.

cal signal within touch-sensitive neurons, in neurites for the transmission of the action potentials from the peripheral neurite to the central synapse, or at the level of the central synapse in transmitter release. In an attempt to discern between these possibilities we attempted to drive motor behaviors in touché mutants optogenetically with channelrhodopsin-2. We found that wild-type and touché mutants expressing channelrhodopsin-2 in sensory neurons responded to blue light $(480 \mathrm{~nm})$ with motor behaviors. These findings indicate that touché mutant sensory neurons can activate second order neurons leading to motor behaviors, suggestive of a defect within sensory neuron activation.
To explore the activation of sensory neurons in vivo we developed and used a novel recording preparation which allowed for electrical and mechanical stimulation of the same peripheral neurite. This preparation revealed that the transduction of an electrical signal from the peripheral neurite to the cell body was intact in both wild-type and touché mutants. Furthermore tactile stimuli applied to the same neurite triggered action potentials detectable within the cell bodies of both wild-type and touché mutants. However, in contrast to wild-type RB cells, we did not observe any touché mutant RB cells wherein mechanically evoked action potentials exhibited generator potentials (type I).

The generator potentials observed in type I zebrafish RB neurons are rapidly adapting with activation at both the onset and offset of a stimulus. When combined with the apparent morphology of zebrafish RB neurons, this suggests that the type I cutaneous mechanoreceptors described here are most similar to mammalian $\mathrm{A} \delta$ "free nerve endings," which in addition to mediating tactile stimuli are thought to communicate thermo and nociceptive stimuli. In support of this comparison are reports suggesting that RB neurons are polymodal in nature, as they express multiple receptors (Kucenas et al., 2003; Prober et al., 2008), which are known to participate in the integration of various sensory stimuli in other vertebrates (Cockayne et al., 2000, 2005; Bautista et al., 2006; Kwan et al., 2006). In contrast, type II neurons which lack generator potentials may represent nociceptive neurons which are responding indirectly to tissue damage rather than directly to tactile stimuli. Further experiments involving calibrated low force probes and conduction velocity assays in zebrafish will provide insight into the comparison with mammalian sensory neurons.

Collectively the findings presented here indicate that touche is required either developmentally for the differentiation/retention of light touch-sensitive neurons, or functionally within a subset of touch-sensitive neurons for the conversion tactile stimuli into action potentials via a generator potential dependent process. Discerning between these two possibilities will require a better understanding of the zebrafish sensory neuron population, insights into the genetic pathways governing the development of sensory neuron subtypes, and ultimately the molecular identification of the touché locus.

\section{References}

Bautista DM, Jordt SE, Nikai T, Tsuruda PR, Read AJ, Poblete J, Yamoah EN, Basbaum AI, Julius D (2006) TRPA1 mediates the inflammatory actions of environmental irritants and proalgesic agents. Cell 124:1269-1282.

Boothby KM, Roberts A (1992a) The stopping response of Xenopus laevis embryos: pharmacology and intracellular physiology of rhythmic spinal neurones and hindbrain neurones. J Exp Biol 169:65-86.

Boothby KM, Roberts A (1992b) The stopping response of Xenopus laevis embryos: behaviour, development and physiology. J Comp Physiol A 170:171-180.

Caron SJ, Prober D, Choy M, Schier AF (2008) In vivo birthdating by BAPTISM reveals that trigeminal sensory neuron diversity depends on early neurogenesis. Development 135:3259-3269.

Clarke JD, Hayes BP, Hunt SP, Roberts A (1984) Sensory physiology, anatomy and immunohistochemistry of Rohon-Beard neurones in embryos of Xenopus laevis. J Physiol 348:511-525.

Cockayne DA, Hamilton SG, Zhu QM, Dunn PM, Zhong Y, Novakovic S, Malmberg AB, Cain G, Berson A, Kassotakis L, Hedley L, Lachnit WG, Burnstock G, McMahon SB, Ford AP (2000) Urinary bladder hyporeflexia and reduced pain-related behaviour in P2X3-deficient mice. Nature 407:1011-1015.

Cockayne DA, Dunn PM, Zhong Y, Rong W, Hamilton SG, Knight GE, Ruan HZ, Ma B, Yip P, Nunn P, McMahon SB, Burnstock G, Ford AP (2005) $\mathrm{P} 2 \mathrm{X} 2$ knockout mice and $\mathrm{P} 2 \mathrm{X} 2 / \mathrm{P} 2 \mathrm{X} 3$ double knockout mice reveal a role 
for the $\mathrm{P} 2 \mathrm{X} 2$ receptor subunit in mediating multiple sensory effects of ATP. J Physiol 567:621-639.

Colbert HA, Bargmann CI (1995) Odorant-specific adaptation pathways generate olfactory plasticity in C. elegans. Neuron 14:803-812.

Douglass AD, Kraves S, Deisseroth K, Schier AF, Engert F (2008) Escape behavior elicited by single, channelrhodopsin-2-evoked spikes in zebrafish somatosensory neurons. Curr Biol 18:1133-1137.

Drapeau P, Ali DW, Buss RR, Saint-Amant L (1999) In vivo recording from identifiable neurons of the locomotor network in the developing zebrafish. J Neurosci Methods 88:1-13.

Drew LJ, Wood JN, Cesare P (2002) Distinct mechanosensitive properties of capsaicin-sensitive and -insensitive sensory neurons. J Neurosci 22:RC228.

Drew LJ, Rohrer DK, Price MP, Blaver KE, Cockayne DA, Cesare P, Wood JN (2004) Acid-sensing ion channels ASIC2 and ASIC3 do not contribute to mechanically activated currents in mammalian sensory neurones. J Physiol 556:691-710.

Granato M, van Eeden FJ, Schach U, Trowe T, Brand M, Furutani-Seiki M, Haffter P, Hammerschmidt M, Heisenberg CP, Jiang YJ, Kane DA, Kelsh RN, Mullins MC, Odenthal J, Nüsslein-Volhard C (1996) Genes controlling and mediating locomotion behavior of the zebrafish embryo and larva. Development 123:399-413.

Haffter P, Nüsslein-Volhard C (1996) Large scale genetics in a small vertebrate, the zebrafish. Int J Dev Biol 40:221-227.

Haffter P, Granato M, Brand M, Mullins MC, Hammerschmidt M, Kane DA, Odenthal J, van Eeden FJ, Jiang YJ, Heisenberg CP, Kelsh RN, FurutaniSeiki M, Vogelsang E, Beuchle D, Schach U, Fabian C, Nüsslein-Volhard C (1996) The identification of genes with unique and essential functions in the development of the zebrafish, Danio rerio. Development 123:1-36.

Hamill OP, Marty A, Neher E, Sakmann B, Sigworth FJ (1981) Improved patch-clamp techniques for high-resolution current recording from cells and cell-free membrane patches. Pflugers Arch 391:85-100.

Higashijima S, Masino MA, Mandel G, Fetcho JR (2003) Imaging neuronal activity during zebrafish behavior with a genetically encoded calcium indicator. J Neurophysiol 90:3986-3997.

Kimmel CB, Ballard WW, Kimmel SR, Ullmann B, Schilling TF (1995) Stages of embryonic development of the zebrafish. Dev Dyn 203:253-310.

Kindt KS, Viswanath V, Macpherson L, Quast K, Hu H, Patapoutian A, Schafer WR (2007) Caenorhabditis elegans TRPA-1 functions in mechanosensation. Nat Neurosci 10:568-577.

Kucenas S, Li Z, Cox JA, Egan TM, Voigt MM (2003) Molecular characterization of the zebrafish P2X receptor subunit gene family. Neuroscience 121:935-945.

Kwan KY, Allchorne AJ, Vollrath MA, Christensen AP, Zhang DS, Woolf CJ, Corey DP (2006) TRPA1 contributes to cold, mechanical, and chemical nociception but is not essential for hair-cell transduction. Neuron 50:277-289.
Liedtke W, Tobin DM, Bargmann CI, Friedman JM (2003) Mammalian TRPV4 (VR-OAC) directs behavioral responses to osmotic and mechanical stimuli in Caenorhabditis elegans. Proc Natl Acad Sci U S A 100 [Suppl 2]:14531-14536.

Moffatt L, Hume RI (2007) Responses of rat P2X2 receptors to ultrashort pulses of ATP provide insights into ATP binding and channel gating. J Gen Physiol 130:183-201.

Nicolson T, Rüsch A, Friedrich RW, Granato M, Ruppersberg JP, NüssleinVolhard C (1998) Genetic analysis of vertebrate sensory hair cell mechanosensation: the zebrafish circler mutants. Neuron 20:271-283.

O'Hagan R, Chalfie M, Goodman MB (2005) The MEC-4 DEG/ENaC channel of Caenorhabditis elegans touch receptor neurons transduces mechanical signals. Nat Neurosci 8:43-50.

Paukert M, Sidi S, Russell C, Siba M, Wilson SW, Nicolson T, Gründer S (2004) A family of acid-sensing ion channels from the zebrafish: widespread expression in the central nervous system suggests a conserved role in neuronal communication. J Biol Chem 279:18783-18791.

Postlethwait JH, Johnson SL, Midson CN, Talbot WS, Gates M, Ballinger EW, Africa D, Andrews R, Carl T, Eisen JS (1994) A genetic linkage map for the zebrafish. Science 264:699-703.

Prober DA, Zimmerman S, Myers BR, McDermott BM Jr, Kim SH, Caron S, Rihel J, Solnica-Krezel L, Julius D, Hudspeth AJ, Schier AF (2008) Zebrafish TRPA1 channels are required for chemosensation but not for thermosensation or mechanosensory hair cell function. J Neurosci 28:10102-10110.

Ribera AB, Nüsslein-Volhard C (1998) Zebrafish touch-insensitive mutants reveal an essential role for the developmental regulation of sodium current. J Neurosci 18:9181-9191.

Saint-Amant L, Drapeau P (1998) Time course of the development of motor behaviors in the zebrafish embryo. J Neurobiol 37:622-632.

Seiler C, Nicolson T (1999) Defective calmodulin-dependent rapid apical endocytosis in zebrafish sensory hair cell mutants. J Neurobiol 41: $424-434$

Sidi S, Friedrich RW, Nicolson T (2003) NompC TRP channel required for vertebrate sensory hair cell mechanotransduction. Science 301:96-99.

Sneddon LU (2003) Trigeminal somatosensory innervation of the head of a teleost fish with particular reference to nociception. Brain Res 972:44-52.

Suzuki M, Mizuno A, Kodaira K, Imai M (2003) Impaired pressure sensation in mice lacking TRPV4. J Biol Chem 278:22664-22668.

Uemura O, Okada Y, Ando H, Guedj M, Higashijima S, Shimazaki T, Chino N, Okano H, Okamoto H (2005) Comparative functional genomics revealed conservation and diversification of three enhancers of the isll gene for motor and sensory neuron-specific expression. Dev Biol 278:587-606.

Walker RG, Willingham AT, Zuker CS (2000) A Drosophila mechanosensory transduction channel. Science 287:2229-2234.

Westerfield M (2000) The zebrafish book. A guide for the laboratory use of zebrafish (Danio rerio), Ed 4. Eugene, OR: University of Oregon. 